\title{
Characteristics of Patients Presenting to a Psycho-Oncology Outpatient Clinic
}

\author{
C. Hyung Keun Park ${ }^{1}$, Harin $\mathrm{Kim}^{2}$, Yangsik $\mathrm{Kim}^{3}$, and Yeon $\mathrm{Ho} \mathrm{Joo}^{2} \bowtie$ \\ ${ }^{1}$ Department of Psychiatry, Asan Medical Center, Seoul, Republic of Korea \\ ${ }^{2}$ Department of Psychiatry, Asan Medical Center, University of Ulsan College of Medicine, Seoul, Republic of Korea \\ ${ }^{3}$ Mental Health Research Institute, National Center for Mental Health, Seoul, Republic of Korea
}

\begin{abstract}
Objective We aimed to determine the overall profile of patients in a psycho-oncology clinic and the differences in their characteristics according to the cancer site.

Methods The charts of 740 patients aged under 81 years were reviewed. The data from 586 completed questionnaires were subjected to multiple comparison analyses using one-way analysis of variance to examine the demographic and clinical differences according to the cancer site.
\end{abstract}

Results Most ( $n=532,71.9 \%)$ patients were referred. Most new patients $(n=426,96.6 \%)$ received a psychiatric diagnosis; the most common diagnosis was depressive disorder $(n=234,31.6 \%)$. Likewise, depressive disorder accounted for the majority of diagnoses in all groups except for the digestive system cancer group in which sleep-wake disorder was the most prevalent. The female genital cancer group showed a higher level of anxiety symptoms than other groups, except for breast and haematolymphoid cancer groups, and psychological distress than all other groups.

Conclusion There appear to be delays in the referral of cancer patients seeking psychiatric help to a psycho-oncology clinic. Along with tailoring approaches by cancer site, thorough evaluation and appropriate management of sleep-wake and anxiety symptoms are important for digestive system and female genital cancer patients, respectively.

Psychiatry Investig 2021;18(8):743-754

Key Words Psycho-oncology, Neoplasms, Outpatients, Anxiety, Psychological distress.

\section{INTRODUCTION}

Cancer is a leading cause of death worldwide, and accounted for approximately 9.6 million deaths in $2018 .{ }^{1}$ In the Republic of Korea (hereafter, Korea), cancer has been the most common cause of death since $1983,{ }^{2}$ accounting for 78,863 deaths (27.6\% of all deaths) in the country in 2017 . The overall incidence rates of all cancers increased annually by about $3.5 \%$ from 1999 to 2011 and then decreased annually by about $2.7 \%$ until 2017. The mortality rates of all cancers also decreased annually since 2002 by $2.8 \%$. Although the overall incidence rates of cancers have been decreasing in recent years, dimin-

Received: March 11, 2021 Revised: May 20, 2021

Accepted: May 27, 2021

$\triangle$ Correspondence: Yeon Ho Joo, MD, PhD

Department of Psychiatry, Asan Medical Center, University of Ulsan College of Medicine, 88 Olympic-ro 43-gil, Songpa-gu, Seoul 05505, Republic of Korea Tel: +82-2-3010-3412, Fax: +82-2-485-8381, E-mail: jooyeonho@gmail.com

(c) This is an Open Access article distributed under the terms of the Creative Commons Attribution Non-Commercial License (https://creativecommons.org/licenses/by$\mathrm{nc} / 4.0$ ) which permits unrestricted non-commercial use, distribution, and reproduction in any medium, provided the original work is properly cited. ishing mortality rates continue to contribute to the improvement of survival rates, and consequently, the annual cancer prevalence rate has increased; by the end of 2017, there were 1.87 million cancer cases nationwide, consisting of $3.6 \%$ of the Korean population. ${ }^{3}$ In terms of disease burden estimated as cause-specific Disability-Adjusted Life Years, cancer accounts for the largest proportion in Korea ${ }^{4}$ and worldwide. ${ }^{5}$

During diagnosis and treatment, cancer patients experience significant psychological problems such as distress, depression, anxiety, post-traumatic stress, and demoralization. ${ }^{6}$ Even after excluding simple psychological distress under diagnostic threshold, psychiatric disorders are diagnosed in about a third of cancer patients, ${ }^{7,8}$ and they are known to have an adverse effect on cancer management. Depression can reduce compliance to treatment and negatively affect the course of cancer, increasing the duration of hospital stay and cost of treatment. ${ }^{9}$ Psychiatric morbidity is associated with elevated mortality. ${ }^{10}$ Therefore, as the number of cancer patients is increasing, it has become important to address their mental illnesses. In response, the field of psycho-oncology emerged in 
the mid-1970s ${ }^{11}$ and encompasses psychological, social, and spiritual aspects of cancer; it now occupies a crucial part in the integrated care of cancer patients. ${ }^{12}$

Psycho-oncology services are offered through inpatient or outpatient settings. To provide optimal interventions, an indepth understanding of the characteristics of the target population is needed. Most studies on patients receiving psychooncology care have involved inpatients ${ }^{13-15}$ or a combined group of inpatients and outpatients. ${ }^{16}$ However, to our knowledge, only two studies have investigated the nature of outpatient psycho-oncology care, where one study included only new patients ${ }^{17}$ and the other assessed only limited aspects (age, sex, performance status, cancer site, and psychiatric diagnosis) of its study sample. ${ }^{18}$ Hence, there is a clear gap in the knowledge on the characteristics of the population in terms of both psycho-oncology care and cancer treatment. Moreover, the previous studies did not assess the severity of psychiatric symptoms. Among individuals with the same psychiatric diagnosis, such as depression or anxiety, the severity of the symptoms could vary. Thus, a study covering all consecutive patients, both new and return, and addressing comprehensive aspects related to the use of psycho-oncology services, cancer treatment, and the degree of psychiatric symptoms is needed to understand the overall picture.

In this exploratory, cross-sectional research, we aimed to determine the overall profile of patients presenting to a psycho-oncology outpatient clinic in a tertiary hospital and the differences in their characteristics according to the primary cancer site for which they were being or had been treated for. A good understanding of their psychiatric needs, in the long term, will equip psycho-oncologists and referring doctors with the measures and ability to provide optimal interventions to this special group. In this work, the term "cancer patients" will refer to patients once diagnosed with cancer regardless of their current cancer treatment status.

\section{METHODS}

\section{Patients}

The Asan Medical Center, located in Seoul, Korea, is a 2,705bed tertiary hospital, providing treatments in all fields of medical and surgical specialties. In its cancer hospital, the Asan Cancer Institute, 917,892 outpatients and 76,380 inpatients received treatment in 2019. ${ }^{19}$ This study was conducted in the Stress Clinic for Cancer Patients, a specialized psycho-oncology outpatient clinic, in the Asan Cancer Institute, where cancer patients above 17 years of age with psychiatric problems were treated by psychiatric specialists. Patients can be referred to or visit the facility on their own. A routine symptom questionnaire is provided as part of the clinical service. Charts of consecutive series of patients' first visits, defined as index visits, to the clinics of the authors (CHKP, HK, YK) in a period of 1 year (from May 21, 2019 to May 20, 2020) were reviewed. Patients who refused to complete the questionnaire, were medically overly unstable, were over 80 years of age, or had difficulty understanding the Korean language were excluded from the survey. As a retrospective chart review, the approval from the Institutional Review Board of Asan Medical Center was obtained (2020-1755), and the need for informed consent was waived.

\section{Measures}

\section{Demographic and visit-related variables}

Psychiatric diagnosis was made according to the Diagnostic and Statistical Manual of Mental Disorders, Fifth Edition (DSM 5) during the index visits by clinical interview with the clinicians (CHKP, HK, YK).

\section{Cancer-related variables}

Index cancer was defined as the cancer dominating treatment during the index visit. Sites of origin of the index cancer were grouped according to the World Health Organization Classification of Tumours, ${ }^{20}$ and the date of index cancer diagnosis, determined according to the hierarchy from the European Network of Cancer Registries, ${ }^{21}$ was used to record the time since index cancer diagnosis. Disease status was categorized as either free or active, based on the criteria developed in a previous study ${ }^{22}$ and modified by the authors. Radiofrequency ablation was added as an alternative treatment to surgery for one of the conditions for disease-free status: "metastatic cancer that had been surgically removed, and no documentation of recurrence." Types of anticancer treatments received during 2 months before the index visit date were recorded: surgery, chemotherapy, radiotherapy, or other therapy (hormone therapy, immunotherapy, and targeted therapy); the duration of interest was adopted from an earlier study. ${ }^{22}$

\section{Psychiatric-related variable: diagnosis}

Psychiatric diagnosis was made according to the Diagnostic and Statistical Manual of Mental Disorders, Fifth Edition $(\mathrm{DSM}-5)^{23}$ during the index visits.

\section{Functional and emotional assessment}

The 27-item self-report Functional Assessment of the Cancer Therapy-General (FACT-G) was used to evaluate various aspects of the health-related quality of life during the past week. $^{24,25}$ The scale consists of four subscales: "physical wellbeing (PWB)," "social/family well-being (SWB)," "emotional well-being (EWB)," and "functional well-being (FWB)," with 
each subscore ranging from 0 to $28,28,24$, and 28 , respectively. A higher subscore corresponds to a higher level of the health-related quality of life.

The 14-item self-report Hospital Anxiety and Depression Scale (HADS), ${ }^{26,27}$ composed of two subscales for depressive and anxiety symptoms, was employed to evaluate their presence and severity during the past week. Each subscore ranges from 0 to 21, with a higher score indicating greater severity, which was treated in two ways. First, each was used to classify patients as either presence (subscore $\geq 8$ ) or absence (subscore $<8$ ) of depressive and anxiety symptoms each. ${ }^{27}$ Second, each was employed as a continuous variable reflecting magnitude.

The 1-item self-report Distress Thermometer (DT) was used to detect psychological distress during the past week. ${ }^{28,29}$ The score ranges from 0 (no distress) to 10 (extreme distress), which was employed in two ways. First, the score of four or more was categorized as clinically significant distress. ${ }^{29}$ Second, the score was regarded as a continuous variable of the severity of distress.

Item 9 of the 9-item self-report Patient Health Questionnaire-9 (PHQ-9), used to assess the nine symptoms of a major depressive episode (Diagnostic and Statistical Manual of Mental Disorders, Fourth Edition), ${ }^{30}$ was employed to address the presence of suicidal ideation for the past two weeks. ${ }^{31,32}$ Its scoring is based on symptom frequency: "not at all," "several days," "more than half the days," or "nearly every day" assigned $0,1,2$, or 3 points, respectively. In the present study, the patients who reported such ideation for at least several days (PHQ-9 Item 9 score $\geq 1$ ) were categorized as having suicidal ideation.

\section{Statistical analyses}

The demographic and clinical variables of all patients were analyzed using descriptive statistics. To evaluate the representativeness of the completers who had filled out the questionnaire, the characteristics of the completers and the non-completers were compared. Student's t-test for continuous variables and Pearson's $\chi^{2}$ test or Fisher's exact test, in case the numbers in the cells were $<5$, were employed for categorical variables.

As a subgroup analysis on the completers, to examine the differences in demographic and clinical factors according to the cancer site, multiple comparison analyses were conducted with each factor across cancer sites using one-way analysis of variance (ANOVA) with Duncan's post-hoc analysis for continuous variables and Pearson's $\chi^{2}$ test or Fisher's exact test for categorical variables. For continuous variables, in case of heterogeneity of variance among cancer sites, Welch's ANOVA and Dunnett's T3 test were used instead. Missing values were excluded from each analysis. All statistical analyses were performed using SPSS for Windows version 21.0 for PC (IBM
Corp., Armonk, NY, USA). A two-tailed p value $<0.050$ was considered statistically significant.

\section{RESULTS}

\section{Demographic and visit-related characteristics}

During the study period, 742 patients aged 80 years or younger visited the psycho-oncology outpatient clinic; two had benign tumors and were excluded from the analysis (Table 1), making the number in our study sample 740 . The ratio of female to male patients was 2.2. Approximately $60 \%$ of the patients were new. The mean time since first visit for the followup patients was 29.6 [standard deviation $(\mathrm{SD})=26.1$ ] months The majority (71.9\%) of the patients had been referred from other departments.

\section{Cancer-related characteristics}

On the average, $33.8(\mathrm{SD}=41.7)$ months had passed since the diagnosis of index cancer. Breast cancer (40.0\%) and digestive system cancer (30.8\%) accounted for the majority of cancer cases. Active disease status was observed in $43.8 \%$ of the patients, and about $70 \%$ had received some type of anticancer treatment during the past 2 months.

\section{Psychiatry-related characteristics}

Regarding psychiatric diagnoses, depressive disorder accounted for the largest proportion of cases (31.6\%), followed by sleep-wake disorder (24.9\%) and adjustment disorder (18.4\%). Mood disorder (depressive disorder and bipolar disorder), sleep-wake disorder, adjustment disorder, anxiety disorder, and delirium (4.5\%) were some of the common diagnoses. When only the new patients were analyzed, most $(n=426)$ had received a psychiatric diagnosis (96.6\%).

\section{Characteristics according to the cancer site: demographic, cancer-related, and psychiatric-related variables}

There was a significant difference in age among index cancer site groups $(\mathrm{F}=23.715, \mathrm{p}<0.001)$ (Table 2); patients in the breast cancer and female genital cancer groups were significantly younger than those in the other cancer site groups, except for miscellaneous cancer.

Time since index cancer diagnosis differed significantly across index cancer sites $(\mathrm{F}=7.821, \mathrm{p}<0.001)$, with the duration since diagnosis of breast cancer being longer than that of digestive system cancer, thorax cancer, and miscellaneous cancer. In the previous 2 months, a large proportion of patients with female genital cancer and miscellaneous cancer (about 50\%) received some type of anticancer treatment.

The distribution of psychiatric diagnoses was significantly 
Table 1. Characteristics of all patients and comparison of the characteristics between non-completers and completers

\begin{tabular}{|c|c|c|c|c|c|c|}
\hline Variables & $\begin{array}{c}\text { Total } \\
(\mathrm{N}=740)\end{array}$ & $\begin{array}{c}\text { Non-completers } \\
\quad(\mathrm{N}=154)\end{array}$ & $\begin{array}{l}\text { Completers } \\
(\mathrm{N}=586)\end{array}$ & $\begin{array}{c}\text { Test statistic } \\
\left(\chi^{2} \text { or } \mathrm{t}\right)\end{array}$ & $\mathrm{p}$ & OR \\
\hline \multicolumn{7}{|l|}{ Demographic } \\
\hline Sex, N (\%) & & & & 9.145 & $0.001^{*}$ & 0.570 \\
\hline Female & $507(68.5)$ & $90(58.4)$ & $417(71.2)$ & & & \\
\hline Male & $233(31.5)$ & $64(41.6)$ & $169(28.8)$ & & & \\
\hline Age, $M(S D), y$ & $56.2(12.0)$ & $59.8(12.5)$ & $55.2(11.7)$ & 4.232 & $<0.001^{*}$ & - \\
\hline \multicolumn{7}{|l|}{ Visit-related } \\
\hline Visit type, N (\%) & & & & 8.475 & $0.004^{*}$ & 0.590 \\
\hline First visit & $441(59.6)$ & $76(49.4)$ & $365(62.3)$ & & & \\
\hline Follow-up visit & $299(40.4)$ & $78(50.6)$ & $221(37.7)$ & & & \\
\hline Time since a first visit ${ }^{\dagger}, \mathrm{M}(\mathrm{SD}), \mathrm{y}$ & $29.6(26.1)$ & $29.8(25.5)$ & $29.6(26.4)$ & 0.072 & 0.943 & - \\
\hline Referral (from), N (\%) & & & & 41.485 & $<0.001^{*}$ & - \\
\hline None & $208(28.1)$ & $75(48.7)$ & $133(22.7)$ & & & \\
\hline Outpatient clinics & $453(61.2)$ & $70(45.5)$ & $383(65.4)$ & & & \\
\hline Inpatient wards & $79(10.7)$ & $9(5.8)$ & $70(11.9)$ & & & \\
\hline \multicolumn{7}{|l|}{ Cancer-related } \\
\hline Index cancer site, $\mathrm{N}(\%)$ & & & & 18.777 & $0.005^{*}$ & - \\
\hline Breast & $296(40.0)$ & $41(26.6)$ & $255(43.5)$ & & & \\
\hline Digestive system & $228(30.8)$ & $53(34.4)$ & $175(29.9)$ & & & \\
\hline Thorax & $64(8.6)$ & $17(11.0)$ & $47(8.0)$ & & & \\
\hline Haematolymphoid & $56(7.6)$ & $18(11.7)$ & $38(6.5)$ & & & \\
\hline Urinary and male genital & $36(4.9)$ & $9(5.8)$ & $27(4.6)$ & & & \\
\hline Female genital & $27(3.6)$ & $5(3.2)$ & $22(3.8)$ & & & \\
\hline Miscellaneous ${ }^{\ddagger}$ & $33(4.5)$ & $11(7.1)$ & $22(3.8)$ & & & \\
\hline Number of cancer diagnoses, N (\%) & & & & - & 0.081 & - \\
\hline 1 & $664(89.7)$ & $132(85.7)$ & $532(90.8)$ & & & \\
\hline 2 & $73(9.9)$ & $22(14.3)$ & $51(8.7)$ & & & \\
\hline 3 & $3(0.4)$ & $0(0.0)$ & $3(0.5)$ & & & \\
\hline Time since index cancer diagnosis, $\mathrm{M}$ (SD), $\mathrm{m}$ & $33.8(41.7)$ & $39.8(48.1)$ & $32.2(39.7)$ & 1.815 & 0.071 & - \\
\hline Disease status, $\mathrm{N}(\%)$ & & & & 0.697 & 0.404 & 0.859 \\
\hline Free & $416(56.2)$ & $82(53.2)$ & $334(57.0)$ & & & \\
\hline Active & $324(43.8)$ & $72(46.8)$ & $252(43.0)$ & & & \\
\hline Surgery $(\leq 2 \mathrm{~m}), \mathrm{N}(\%)$ & & & & 0.762 & 0.383 & 1.298 \\
\hline No & $653(88.2)$ & $139(90.3)$ & $514(87.7)$ & & & \\
\hline Yes & $87(11.8)$ & $15(9.7)$ & $72(12.3)$ & & & \\
\hline Chemotherapy ( $\leq 2 \mathrm{~m}), \mathrm{N}(\%)$ & & & & 5.556 & $0.018^{*}$ & 1.615 \\
\hline No & $493(66.7)$ & $115(74.7)$ & $378(64.6)$ & & & \\
\hline Yes & $246(33.3)$ & $39(25.3)$ & $207(35.4)$ & & & \\
\hline Radiotherapy ( $\leq 2 \mathrm{~m}), \mathrm{N}(\%)$ & & & & 1.720 & 0.190 & 1.665 \\
\hline No & $683(92.3)$ & $146(94.8)$ & $537(91.6)$ & & & \\
\hline Yes & $57(7.7)$ & $8(5.2)$ & $49(8.4)$ & & & \\
\hline Other therapy ${ }^{\S}(\leq 2 \mathrm{~m}), \mathrm{N}(\%)$ & & & & 3.142 & 0.076 & 1.408 \\
\hline No & $462(62.7)$ & $106(68.8)$ & $356(61.1)$ & & & \\
\hline Yes & $275(37.3)$ & $48(31.2)$ & $227(38.9)$ & & & \\
\hline
\end{tabular}


Table 1. Characteristics of all patients and comparison of the characteristics between non-completers and completers (continued)

\begin{tabular}{|c|c|c|c|c|c|c|}
\hline Variables & $\begin{array}{c}\text { Total } \\
(\mathrm{N}=740)\end{array}$ & $\begin{array}{c}\text { Non-completers } \\
(\mathrm{N}=154)\end{array}$ & $\begin{array}{c}\text { Completers } \\
(\mathrm{N}=586)\end{array}$ & $\begin{array}{c}\text { Test statistic } \\
\left(\chi^{2} \text { or } \mathrm{t}\right)\end{array}$ & $\mathrm{p}$ & OR \\
\hline Any anticancer therapy" ( $\leq 2 \mathrm{~m}), \mathrm{N}(\%)$ & & & & 14.395 & $<0.001^{*}$ & 2.022 \\
\hline No & $224(30.4)$ & $66(42.9)$ & $158(27.1)$ & & & \\
\hline Yes & $514(69.6)$ & $88(57.1)$ & $426(72.9)$ & & & \\
\hline \multicolumn{7}{|l|}{ Psychiatry-related } \\
\hline Diagnosis, N (\%) & & & & 45.872 & $<0.001^{*}$ & - \\
\hline Depressive disorder & $234(31.6)$ & $59(38.3)$ & $175(29.9)$ & & & \\
\hline Sleep-wake disorder & $184(24.9)$ & $28(18.2)$ & $156(26.6)$ & & & \\
\hline Adjustment disorder & $136(18.4)$ & $12(7.8)$ & $124(21.2)$ & & & \\
\hline Anxiety disorder & $61(8.2)$ & $14(9.1)$ & $47(8.0)$ & & & \\
\hline Delirium & $33(4.5)$ & $18(11.7)$ & $15(2.6)$ & & & \\
\hline Bipolar disorder & $32(4.3)$ & $5(3.2)$ & $27(4.6)$ & & & \\
\hline Miscellaneous $\pi$ & $45(6.1)$ & $15(9.7)$ & $30(5.1)$ & & & \\
\hline None & $15(2.0)$ & $3(1.9)$ & $12(2.0)$ & & & \\
\hline
\end{tabular}

* significant findings at $\mathrm{p}<0.05$, tin case of a follow-up visit, thead and neck (10), central nervous system (8), endocrine (5), soft tissue and bone (5), skin (3), eye (1), adenocarcinoma of unknown primary site (1), \$includes hormone therapy, immunotherapy, and targeted therapy, "includes surgery, chemotherapy, radiotherapy, and other therapy, "somatic symptom disorder (15), substance use disorder (12), other mental disorder (8), psychotic disorder (4), posttraumatic stress disorder (2), obsessive-compulsive disorder (2), intellectual disability (1), neurocognitive disorder (1). OR: odds ratio, M: mean, SD: standard deviation

different among index cancer sites ( $\mathrm{p}<0.001)$. In most groups, depressive disorder was the most common diagnosis; however, for digestive system cancer patients, sleep-wake disorder (30.7\%) was slightly more prevalent than depressive disorder (28.0\%). Breast cancer patients with bipolar disorder accounted for 6.6\% of cases in the cancer site group and 59.4\% (19/32) of all patients with the diagnosis. For urinary and male genital cancer patients, almost $20 \%$ were diagnosed with delirium, whereas in all other groups, less than $10 \%$ received this diagnosis.

\section{Characteristics according to the cancer site: functional and emotional assessment}

In total, 586 (79.2\%) patients completed the symptom questionnaire. Differences in demographic and clinical characteristics between the non-completers and completers are presented in Table 1 . The difficulty in evaluating male patients, older patients, new patients, and patients undergoing anticancer treatment was mirrored in statistically significant differences between the groups. Although the exact reasons they did not complete the questionnaire and the respective percentages were unavailable, from our clinical experiences, we can say that most of them were overly unstable medically, were cognitively or visually impaired, or had difficulty holding a pen because of severe hand tremor or weakness.

A series of ANOVAs indicated significant differences in the HADS Anxiety score $(\mathrm{F}=2.313, \mathrm{p}=0.033)$ and the $\mathrm{DT}(\mathrm{F}=$ $2.294, p=0.034$ ) among the index cancer site groups. The fe- male genital cancer group showed more severe anxiety symptoms than all other groups, except for breast and haematolymphoid cancer groups, and higher psychological distress than all cancer site groups.

\section{DISCUSSION}

In the present study, we presented a comprehensive description of the characteristics of patients presenting to the psycho-oncology outpatient clinic over a 1-year period. Most patients initially visited the clinic after referral. Moreover, most new patients received a psychiatric diagnosis, and depressive disorder accounted for the largest percentage, with sleepwake disorder and adjustment disorder being the second and the third most prevalent diagnoses, respectively. Likewise, depressive disorder accounted for the majority of diagnoses in all groups by cancer site except for the group of digestive system cancer, in which sleep-wake disorder was prevalent. Female genital cancer patients showed the highest severity of anxiety symptoms and psychological distress.

The female predominance and average age of our study sample were similar to the results of two previous studies which reported these as $62 \%$ and $67 \%$ and $51.22 \pm 14.55$ and $59 \pm 13$ years, respectively. ${ }^{17,18}$ In 2017, of all the cancer patients in Korea, $56.1 \%$ were female (the number of female and male patients were $1,047,567$ and 819,838 , respectively). ${ }^{33}$ The female majority in our study sample and the higher proportion of women in the sample than in the entire cancer patients can be 


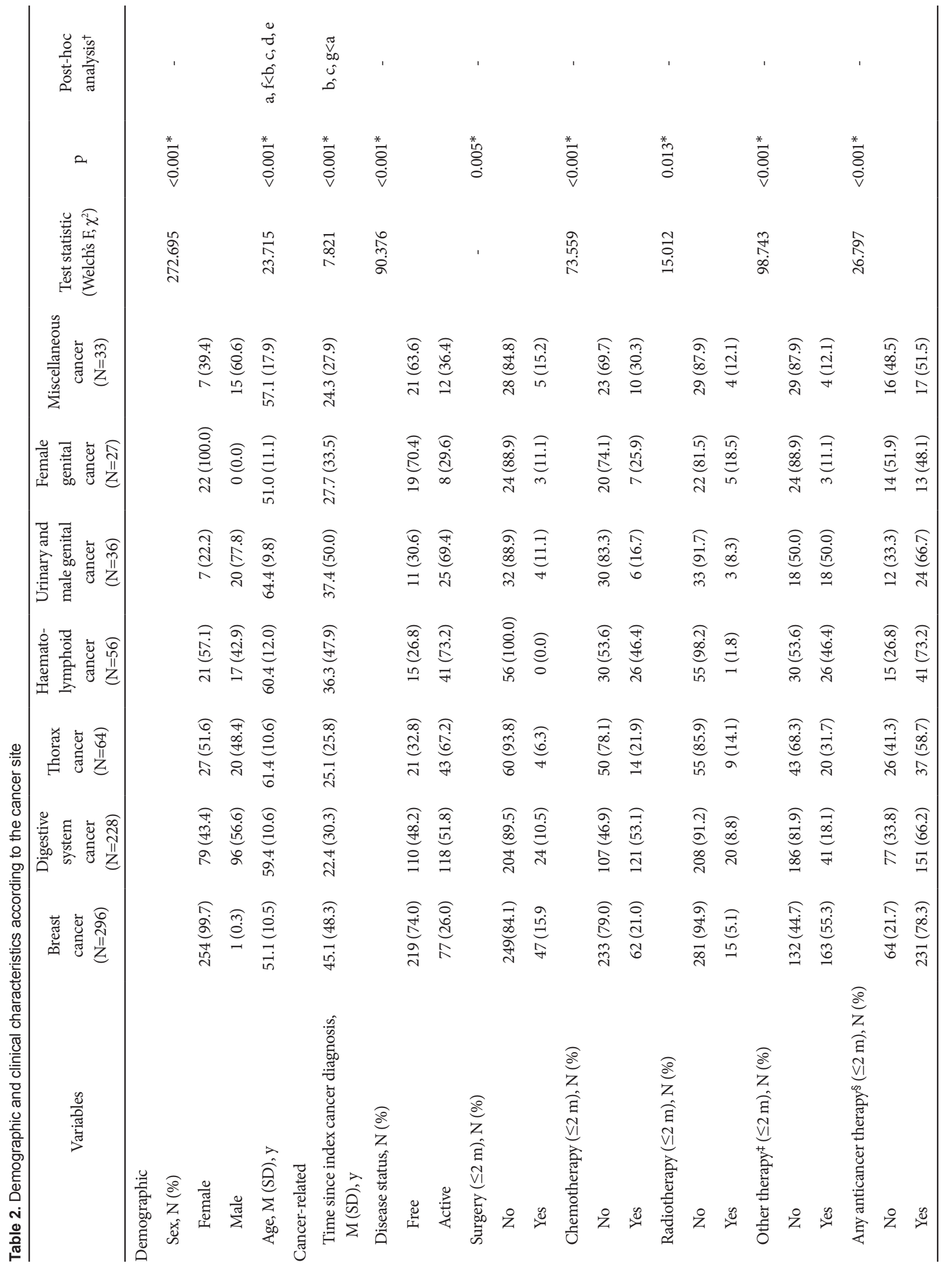




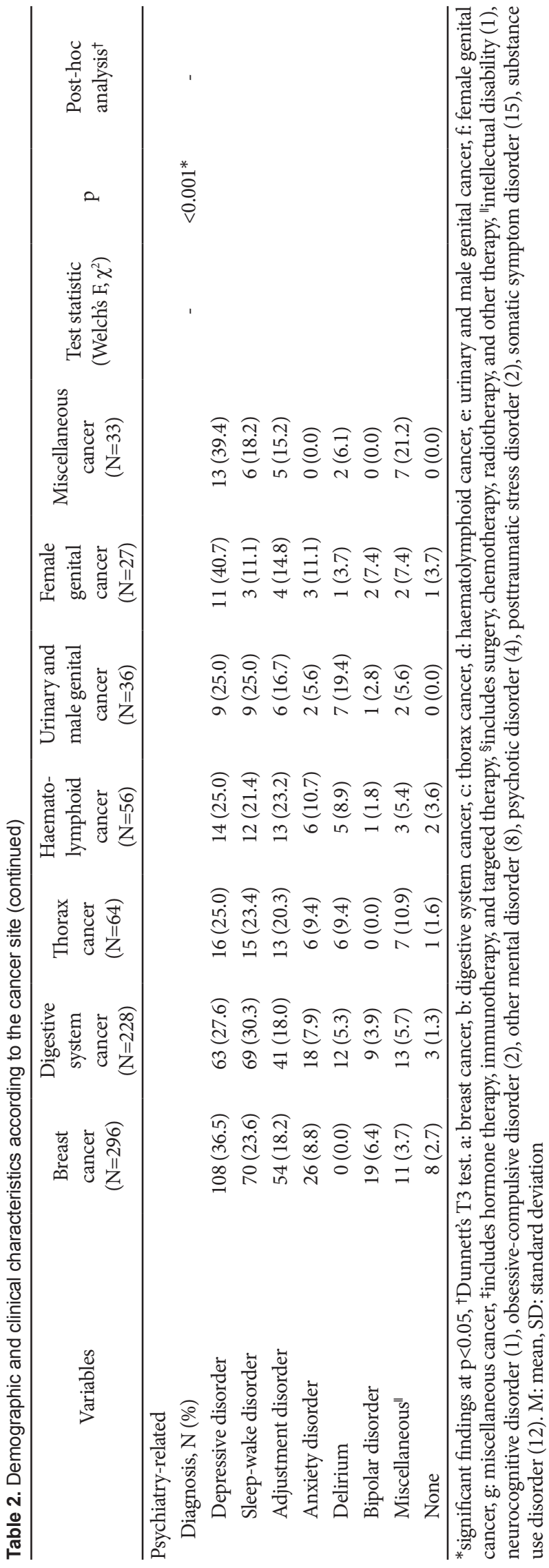

explained in the following ways. First, breast cancer accounted for the largest proportion of cancer cases in our sample. Second, women are more likely to use mental health services than men, ${ }^{34,35}$ and a similar phenomenon is observed among cancer patients. ${ }^{36}$ Third, most of the patients had psychiatric disorder documented to be more prevalent in women-depressive disorder, ${ }^{37}$ insomnia disorder, ${ }^{38}$ and anxiety disorder. ${ }^{39}$

Most patients were initially referred by doctors in other departments. The referred proportion (71.9\%) was lower than that $(83.5 \%)$ in a previous study ${ }^{17}$; setting an ideal proportion of referrals seems unnecessary because, after all, the only thing that matters is to ensure that as many help-seeking patients as possible receive appropriate and timely psycho-oncology care. Therefore, with the statistics being checked regularly, if the proportion is close to $100 \%$, providing patients in oncology clinics with information on psycho-oncology services and ensuring accessibility could be important. Considering some patients' reluctance in disclosing their psychological distress, ${ }^{40}$ a depression self-screening system for cancer patients using touch-screen kiosks at an oncology clinic ${ }^{41}$ could be a practical option because voluntary screening is believed to be efficient, has a high positive predictive value, ${ }^{42}$ and can reduce the risk of nocebo effect. ${ }^{43}$ On the contrary, in case of declining proportion, promoting oncologists' awareness of psychological distress of their patients and psycho-oncology services might be necessary. Regarding oncologists' tendency to avoid addressing psychological issues, ${ }^{44}$ undertraining in detecting psychological symptoms, and insufficient consultation time, ${ }^{45}$ education on psychological distress and training for efficient communication skills to discuss the issues would be helpful.

Among digestive system cancer patients, 101 (13.64\%) had gastrointestinal cancer (data not shown), similar to the finding of a previous study. ${ }^{17}$ However, the distribution of the cancer sites in our study sample was different from that in the general population, especially thyroid, breast, and haematolymphoid cancer. Thyroid cancer, the most common cancer both in females (31.9\%) and in the whole population (21.7\%), accounted for a minimal proportion in our study sample $(\mathrm{n}=$ 4). This seemingly unexpected finding can be explained as follows: This cancer type is well-known for its overall excellent prognosis. ${ }^{46}$ The lowest prevalence of major depressive disorder has been reported in thyroid cancer patients among those with the top 10 most common cancers in Korea because patients regard this cancer type as treatable and curable. ${ }^{47}$ On the other hand, breast cancer patients are well-known to have significant psychological distress. They can undergo distress from treatment, fear of recurrence, problems with body image and sexuality, in addition to side effects of chemotherapy and endocrine therapy. ${ }^{48}$ It was reported that $45 \%$ and $42 \%$ of earlystage and metastatic breast cancer patients, respectively, have 
a psychiatric diagnosis, and about a third of each group has a mood disorder. ${ }^{49}$ Especially, young breast cancer patients under 40 years of age have an elevated risk of psychosocial burden on their life stage-related tasks such as fertility preservation and family planning, developing careers, and nurturing young children. ${ }^{50}$ Haematolymphoid cancer was more common than urinary and male/female genital cancer. In the aforementioned study, patients with non-Hodgkin's lymphoma, the most common type of haematolymphoid cancer, ${ }^{33}$ showed the second highest prevalence of major depressive disorder after patients with lung cancer, ${ }^{47}$ which may partly be explained by the overrepresentation of the cancer site group in our study sample.

The vast majority of new patients $(96.6 \%)$ were diagnosed with a psychiatric disorder, indicating that they saw psychiatrists once they had fully developed the disorder. Previous studies have reported similar numbers [e.g., $97.5 \%{ }^{17}$ and $82.9 \%$ $\left.(316 / 381)^{18}\right]$. Thus, there may be many cancer patients with undiagnosed psychiatric disorders in other departments. Considering the degree of reluctance from both patients and doctors in discussing the psychiatric symptoms ${ }^{40,44}$ and insufficient length of consultation in busy oncology clinics, promptly recognizing and comfortably discussing emotional distress might help minimize the delays in referrals. Moreover, besides full-blown psychiatric disorders, even subthreshold psychological distress appears to negatively affect the quality of life. Haematological cancer patients with subsyndromal depression show no difference in their quality of life compared to those with major depressive disorder. ${ }^{51} \mathrm{~A}$ study on patients with head and neck cancer reported that timely intervention using psychotherapy such as cognitive-behavioral therapy reduces the psychological distress-anxiety and depressive symptoms-at sub-clinical or clinical levels. ${ }^{52}$ It is noteworthy that our sample had a distinctively high proportion of patients with sleep-wake disorder. ${ }^{17,18}$ During the chart review, we found out that some of the patients who had started treatment in the Sleep Clinic for Cancer Patients, a separate psycho-oncology outpatient service, later, presented to the Stress Clinic for Cancer Patients. Perhaps, the Sleep Clinic for Cancer Patients promoted the recognition and treatment of sleep-wake problems in cancer patients, and some patients there changed their psychiatrists because of various reasons (e.g., scheduling two appointments-psycho-oncologic and oncologic-on the same day), eventually contributing to the high proportion of sleepwake disorder patients.

A longer duration since index cancer diagnosis was observed for patients with breast cancer than for those with digestive system or thorax cancer (Table 2). Considering the 10 most common cancer sites, breast cancer was among the top three cancers with favorable 5-year survival rates whereas pan- creas, gallbladder and bile duct cancer, and lung cancer were the last three. ${ }^{33}$ In addition, breast and female genital cancer patients had a significantly lower mean age than other cancer patients, indicating that breast cancer is the second most common site for the 35-65-year age group in Korea. ${ }^{33}$

There are some noteworthy findings with regards to psychiatric diagnosis by index cancer site. Patients with digestive system cancer accounted for the highest proportion of sleepwake disorder cases. One study reported that breast cancer patients showed the highest prevalence of insomnia. ${ }^{53}$ This discrepancy can be attributed to the different characteristics of the study samples and our inclusion of not only insomnia but also other diagnoses belonging to DSM-5 sleep-wake disorder category. This finding suggests that thorough evaluation of sleep-wake problems should be carried out in patients with this cancer type. Moreover, doctors must be encouraged to be equipped with basic, but practical, knowledge on sleep hygiene and use of common hypnotics to reduce distress until their patients can meet a psycho-oncologist. Patients with urinary and male genital cancer accounted for the highest proportion of delirium cases, at nearly $20 \%$. Only the prostate cancer group showed a statistically significant increase in the risk of delirium; prostate cancer patients are 20 times more likely to develop delirium than stomach cancer patients. ${ }^{54}$ Referring doctors must be able to initiate non-pharmacological anti-delirium intervention and use common antipsychotics to control aggressive behavior beyond identifying and treating underlying conditions, which is their expertise, to help prevent injurious events such as falls, which are associated with delirium..$^{55}$ Although only $6.6 \%$ and $7.7 \%$ of the patients with breast cancer and female genital cancer, respectively, were diagnosed with bipolar disorder, they accounted for $65.6 \%$ of all bipolar disorder patients in the sample. Considering no sex difference in the prevalence of the disorder, ${ }^{56}$ the high prevalence of bipolar disorder among patients with breast and female genital cancer, both of which shows a female predominance, could be explained as follows. First, females are more likely to seek professional help with their depressive symptoms than males; thus, they have a higher chance of being diagnosed with mood disorders, including bipolar disorder. ${ }^{57}$ Second, for both bipolar I and II disorder, lifetime prevalence decreases with advancing age group, ${ }^{56}$ and the patients in these the two cancer site groups were significantly younger than those in other groups. Bipolar disorder is long known to be under-diagnosed. ${ }^{58}$ When cancer patients complain of depressive symptoms, if psychooncologists regard their distress simply as a reaction to cancer diagnosis or treatment, the diagnosis of bipolar disorder can be missed. We suggest that bipolar disorder be ruled out before making a diagnosis of unipolar depression, particularly in patients with cancers that occur primarily in women. 
In the subgroup analysis of the questionnaire completers, it turned out to be difficult to assess new and old male patients receiving anticancer treatment (Table 1); an earlier study reported a similar tendency. ${ }^{22}$ The levels of physical, social/family, emotional, and functional well-being and the distribution of the presence or absence of depressive symptoms, anxiety symptoms, psychological distress, and suicidal ideation did not differ among the index cancer site groups (Table 3). These results are understandable as most of our patients received a psychiatric diagnosis; it is likely that the severity of their distress was above the clinical threshold.

Patients with female genital cancer showed higher levels of anxiety symptoms and psychological distress than patients from most other groups. These findings are remarkable considering that this group had a higher proportion of free disease status and lower proportion of recent anticancer treatment than other groups. The results are in line with previous findings; gynecologic cancer patients have a higher level of unadjusted emotional distress than prostate cancer patients, ${ }^{59}$ and ovarian cancer patients are more likely to have clinically significant unadjusted psychological distress than bowel cancer patients. ${ }^{60}$ Female genital cancer has grave, and unique, consequences. Previous researchers speculated that pelvic surgery causes higher psychological distress than other operations, ${ }^{61,62}$ making many patients, who are waiting for their treatment or adapting to the results, depressed and anxious. ${ }^{63}$ Some patients even refuse to undergo surgery such as pelvic evisceration owing to worries about the changes in body image and lifestyle after the operation. ${ }^{63}$ All types of female genital cancers have the possibility of exerting a negative impact on sexual functioning and fertility. ${ }^{64}$ Besides, in ovarian cancer patients, the prevalence of anxiety rather increases after anticancer treatment; preoccupations with poor prognosis of the cancer -death-occur. ${ }^{65}$ Interestingly, along with distress, only anxiety symptoms, not depressive symptoms, were significantly high in female genital cancer patients. These findings correspond with the results of earlier studies that reported a relationship between distress and anxiety or depressive symptoms. ${ }^{66,67}$ Conceptually, psychological distress involves emotional suffering, including depression and anxiety, ${ }^{29,68}$ associated with stressors and burdens difficult to manage in everyday life. ${ }^{68}$ However, distress is more closely related to anxiety than depression in cancer patients ${ }^{66,67}$ As a clinical implication, additional attention to anxiety symptoms should be given in cases of patients with a high distress score.

This study has several limitations. First, due to its cross-sectional design, the causal relationship could not be established. Second, since the degree of psychological difficulties may vary by the stage of disease and treatment status in the cancer population, the present findings cannot be generalized as a uni-

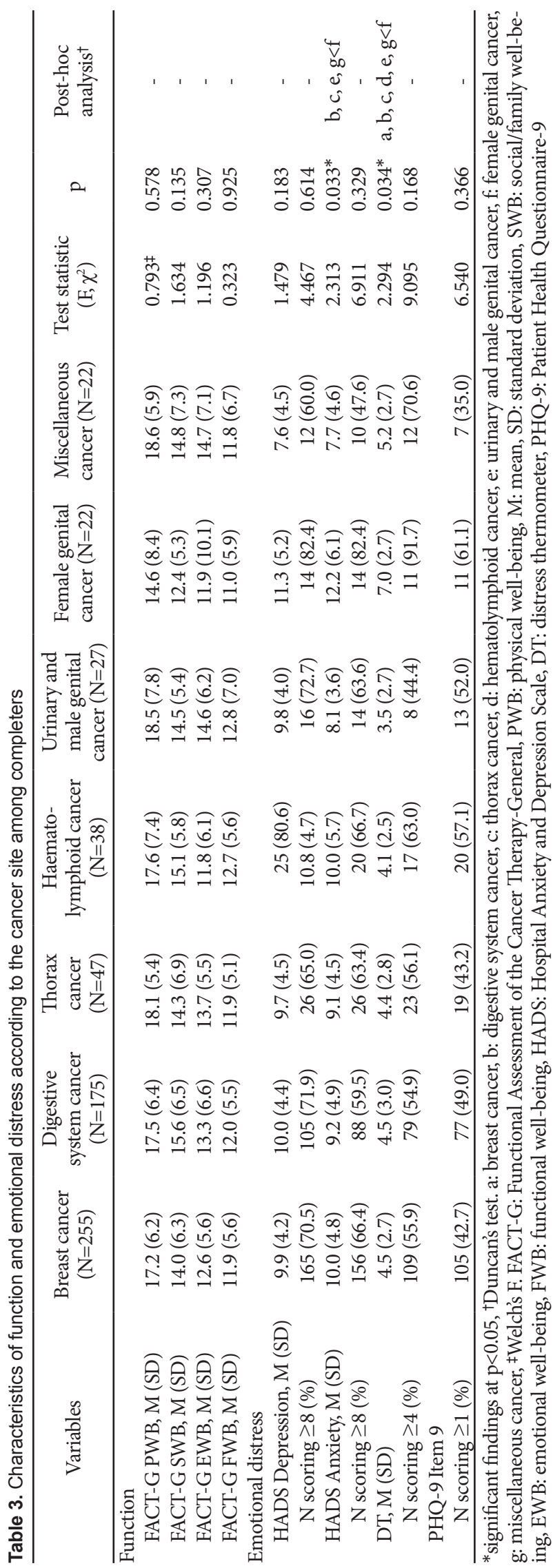


versal feature of each cancer site group. Instead, they should be regarded as characteristics of patients visiting a psychooncology outpatient clinic in a tertiary cancer center. Third, a formal interview instrument for psychiatric diagnosis was not employed, which makes the diagnostic accuracy questionable. However, diagnosis was made entirely by psychiatric specialists with additional training on consultation-liaison psychiatry, and similar diagnoses were grouped to minimize diagnostic uncertainty. Fourth, our study sample consisted of ethnic Koreans only, and thus, generalization of the current findings to other ethnic groups should be performed with caution. Fifth, the present study was performed at one institution, which might have led to institutional bias. Thus, the present results could be more generalizable after multi-institutional studies. Sixth, information on the cancer stage groups (0, I, II, III, or IV) was unavailable from the record except for the TNM stage. Despite the limitations, our study is unique in that we examined multifaceted aspects of the sample including psycho-oncology, anticancer treatment, and quantified psychiatric symptoms.

This cross-sectional study showed the distribution of the patients aged 80 years or younger presenting to a psycho-oncology clinic in a tertiary hospital during 1 year and the differences in their characteristics according to the cancer site. Our findings direct attention to the delays in referral of cancer patients seeking psychiatric help. In addition, tailored approaches by cancer site appear desirable. Thorough evaluation and appropriate management of sleep-wake problems and anxiety symptoms are needed, particularly for digestive system and female genital cancer patients, respectively. Future studies using a diagnostic interview instrument are needed to confirm these findings in a separate sample.

\section{Availability of Data and Material}

The datasets generated or analyzed during the study are available from the corresponding author on reasonable request.

\section{Conflicts of Interest}

The authors have no potential conflicts of interest to disclose.

\section{Author Contributions}

Conceptualization: C. Hyung Keun Park, Yeon Ho Joo. Data curation: Harin Kim. Formal analysis: C. Hyung Keun Park. Investigation: C. Hyung Keun Park, Harin Kim, Yangsik Kim. Methodology: C. Hyung Keun Park. Project administration: C. Hyung Keun Park. Supervision: Yeon Ho Joo. Writing_original draft: C. Hyung Keun Park. Writing-review and editing: all authors.

\section{ORCID iDs}
C. Hyung Keun Park
Harin Kim
Yangsik Kim
Yeon Ho Joo
https://orcid.org/0000-0002-2568-1426 https://orcid.org/0000-0002-7304-4616 https://orcid.org/0000-0003-1841-727X https://orcid.org/0000-0003-0073-8534

\section{Funding Statement None.}

\section{REFERENCES}

1. World Health Organization. Cancer: Key facts. Available at: https:// www.who.int/news-room/fact-sheets/detail/cancer. Accessed March $11,2021$.

2. Korea Statistics. Available at: http://kosis.kr. Accessed March 11, 2021.

3. Hong S, Won YJ, Park YR, Jung KW, Kong HJ, Lee ES. Cancer statistics in Korea: incidence, mortality, survival, and prevalence in 2017. Cancer Res Treat 2020;52:335-350.

4. Yoon SJ, Bae SC, Lee SI, Chang H, Jo HS, Sung JH, et al. Measuring the burden of disease in Korea. J Korean Med Sci 2007;22:518-523.

5. World Health Organization. Global Health Estimates 2016: disease burden by cause, age, sex, by country and by region, 2000-2016. Available at: https://www.who.int/healthinfo/global_burden_disease/estimates/en/index1.html. Accessed March 11, 2021.

6. Grassi L, Spiegel D, Riba M. Advancing psychosocial care in cancer patients. F1000Res 2017;6:2083.

7. Singer S, Das-Munshi J, Brähler E. Prevalence of mental health conditions in cancer patients in acute care--a meta-analysis. Ann Oncol 2010; 21:925-930.

8. Mehnert A, Brähler E, Faller H, Härter M, Keller M, Schulz H, et al. Fourweek prevalence of mental disorders in patients with cancer across major tumor entities. J Clin Oncol 2014;32:3540-3546.

9. Hardman A, Maguire P, Crowther D. The recognition of psychiatric morbidity on a medical oncology ward. J Psychosom Res 1989;33:235239.

10. Wikman A, Ljung R, Johar A, Hellstadius Y, Lagergren J, Lagergren P. Psychiatric morbidity and survival after surgery for esophageal cancer: a population-based cohort study. J Clin Oncol 2015;33:448-454.

11. Holland JC. History of psycho-oncology: overcoming attitudinal and conceptual barriers. Psychosom Med 2002;64:206-221.

12. Lee HJ, Lee KM, Jung D, Shim EJ, Hahm BJ, Kim JH. Psycho-oncology in Korea: past, present and future. Biopsychosoc Med 2017;11:12.

13. Kissane DW, Smith GC. Consultation-liaison psychiatry in an Australian oncology unit. Aust N Z J Psychiatry 1996;30:397-404.

14. Citero Vde A, Nogueira-Martins LA, Lourenço MT, Andreoli SB. Clinical and demographic profile of cancer patients in a consultation-liaison psychiatric service. Sao Paulo Med J 2003;121:111-116.

15. Grassi L, Gritti P, Rigatelli M, Gala C. Psychosocial problems secondary to cancer: an Italian multicentre survey of consultation-liaison psychiatry in oncology. Italian Consultation-Liaison Group. Eur J Cancer 2000; 36:579-585.

16. Akechi T, Nakano T, Okamura H, Ueda S, Akizuki N, Nakanishi T, et al. Psychiatric disorders in cancer patients: descriptive analysis of 1721 psychiatric referrals at two Japanese cancer center hospitals. Jpn J Clin Oncol 2001;31:188-194.

17. Anuk D, Özkan M, Kizir A, Özkan S. The characteristics and risk factors for common psychiatric disorders in patients with cancer seeking help for mental health. BMC Psychiatry 2019;19:269.

18. Tada Y, Matsubara M, Kawada S, Ishida M, Wada M, Wada T, et al. Psychiatric disorders in cancer patients at a university hospital in Japan: descriptive analysis of 765 psychiatric referrals. Jpn J Clin Oncol 2012;42: 183-188.

19. Asan Cancer Institute. Annual Report 2019. Available at: http://cancer. amc.seoul.kr/asan/depts/cancer/K/noticeDetail.do?pageIndex $=1 \&$ me nuId $=3577 \&$ contentId $=4371 \&$ search Condition=\&searchKeyword $=$. Accessed March 11, 2021.

20. World Health Organization. WHO Classification of Tumours. Available at: https://whobluebooks.iarc.fr/. Accessed March 11, 2021.

21. European Network of Cancer Registries. Standards and Guidelines for Cancer Registration in Europe. Available at: https://publications.iarc. fr/_publications/media/download/3779/e5a42821d6e095960a2ea21775b6928de4a616c8.pdf. Accessed March 11, 2021.

22. Walker J, Waters RA, Murray G, Swanson H, Hibberd CJ, Rush RW, et al. Better off dead: suicidal thoughts in cancer patients. J Clin Oncol 
2008;26:4725-4730

23. American Psychiatric Association. Diagnostic and Statistical Manual of Mental Disorders. 5th Ed. Washington: American Psychiatric Publishing; 2013.

24. Cella DF, Tulsky DS, Gray G, Sarafian B, Linn E, Bonomi A, et al. The Functional Assessment of Cancer Therapy scale: development and validation of the general measure. J Clin Oncol 1993;11:570-579.

25. Kim H, Yoo HJ, Kim YJ, Han OS, Lee KH, Lee JH, et al. Development and validation of Korean functional assessment cancer therapy-general (FACT-G). Korean J Clin Psychol 2003;22:215-229.

26. Zigmond AS, Snaith RP. The hospital anxiety and depression scale. Acta Psychiatr Scand 1983;67:361-370.

27. Oh SM, Min KJ, Park DB. A study on the standardization of the hospital anxiety and depression scale for Koreans: a comparison of normal, depressed and anxious groups. J Korean Neuropsychiatr Assoc 1999;38: 289-296.

28. National Comprehensive Cancer Network. Distress management. clinical practice guidelines. J Natl Compr Canc Netw 2003;1:344-374.

29. Shim EJ, Shin YW, Jeon HJ, Hahm BJ. Distress and its correlates in Korean cancer patients: pilot use of the distress thermometer and the problem list. Psychooncology 2008;17:548-555.

30. American Psychiatric Association. Diagnostic and Statistical Manual of Mental Disorders. 4th Ed. Washington: American Psychiatric Association; 1994.

31. Spitzer RL, Kroenke K, Williams JB. Validation and utility of a self-report version of PRIME-MD: the PHQ primary care study. Primary Care Evaluation of Mental Disorders. Patient Health Questionnaire. JAMA 1999;282:1737-1744.

32. An J, Seo E, Lim K, Shin J, Kim J. Standardization of the Korean version of screening tool for depression (Patient Health Questionnaire-9, PHQ9). J Korean Soc Biol Ther Psychiatry 2013;19:47-56.

33. National Cancer Center. Annual report of cancer statistics in Korea in 2017. Available at: https://ncc.re.kr/cancerStatsView.ncc?bbsnum $=518$ $\&$ searchKey=total\&searchValue=\&pageNum=1. Accessed March 11, 2021.

34. Wang PS, Lane M, Olfson M, Pincus HA, Wells KB, Kessler RC. Twelvemonth use of mental health services in the United States: results from the National Comorbidity Survey Replication. Arch Gen Psychiatry 2005; 62:629-640.

35. Park JE, Cho SJ, Lee JY, Sohn JH, Seong SJ, Suk HW, et al. Impact of stigma on use of mental health services by elderly Koreans. Soc Psychiatry Psychiatr Epidemiol 2015; 50: 757-766.

36. Merckaert I, Libert Y, Messin S, Milani M, Slachmuylder JL, Razavi D. Cancer patients' desire for psychological support: prevalence and implications for screening patients' psychological needs. Psychooncology 2010; 19:141-149.

37. Salk RH, Hyde JS, Abramson LY. Gender differences in depression in representative national samples: meta-analyses of diagnoses and symptoms. Psychol Bull 2017;143:783-822.

38. Krishnan V, Collop NA. Gender differences in sleep disorders. Curr Opin Pulm Med 2006;12:383-389.

39. McLean CP, Asnaani A, Litz BT, Hofmann SG. Gender differences in anxiety disorders: prevalence, course of illness, comorbidity and burden of illness. J Psychiatr Res 2011;45:1027-1035.

40. Okuyama T, Endo C, Seto T, Kato M, Seki N, Akechi T, et al. Cancer patients' reluctance to disclose their emotional distress to their physicians: a study of Japanese patients with lung cancer. Psychooncology 2008;17: 460-465.

41. Lee JY, Jung D, Kim WH, Lee HJ, Noh DY, Hahm BJ. Correlates of oncologist-issued referrals for psycho-oncology services: what we learned from the electronic voluntary screening and referral system for depression (eVSRS-D). Psychooncology 2016;25:170-178.

42. Magruder KM, Norquist GS, Feil MB, Kopans B, Jacobs D. Who comes to a voluntary depression screening program? Am J Psychiatry 1995;11: 1615-1622.
43. Thombs BD, Coyne JC, Cuijpers P, De Jonge P, Gilbody S, Ioannidis JP, et al. Rethinking recommendations for screening for depression in primary care. CMAJ 2012;184:413-418.

44. Passik SD, Dugan W, McDonald MV, Rosenfeld B, Theobald DE, Edgerton S. Oncologists' recognition of depression in their patients with cancer. J Clin Oncol 1998;16:1594-1600.

45. Fallowfield L, Ratcliffe D, Jenkins V, Saul J. Psychiatric morbidity and its recognition by doctors in patients with cancer. Br J Cancer 2001;84: 1011-1015.

46. Shaha AR. Implications of prognostic factors and risk groups in the management of differentiated thyroid cancer. Laryngoscope 2004;114:393402.

47. Park B, Youn S, Yi KK, Lee SY, Lee JS, Chung S. The prevalence of depression among patients with the top ten most common cancers in South Korea. Psychiatry Investig 2017;14:618-625.

48. Ganz PA. Psychological and social aspects of breast cancer. Oncology (Williston Park) 2008;22:642-646.

49. Kissane DW, Grabsch B, Love A, Clarke DM, Bloch S, Smith GC. Psychiatric disorder in women with early stage and advanced breast cancer: a comparative analysis. Aust N Z J Psychiatry 2004;38:320-326.

50. Freedman RA, Partridge AH. Management of breast cancer in very young women. Breast 2013;22:S176-S179.

51. Rezaei O, Sharifian RA, Soleimani M, Jahanian A. The quality of life of hematological malignancy patients with major depressive disorder or subsyndromal depression. Int J Psychiatry Med 2012;44:149-163.

52. Kangas M, Milross C, Taylor A, Bryant RA. A pilot randomized controlled trial of a brief early intervention for reducing posttraumatic stress disorder, anxiety and depressive symptoms in newly diagnosed head and neck cancer patients. Psychooncology 2013;22:1665-1673.

53. Davidson JR, MacLean AW, Brundage MD, Schulze K. Sleep disturbance in cancer patients. Soc Sci Med 2002;54:1309-1321.

54. Ko HJ, Youn CH, Chung SE, Kim A, Kim HM. Risk factors related to development of delirium in hospice patients. Korean J Hosp Palliat Care 2014;17:170-178.

55. Lakatos BE, Capasso V, Mitchell MT, Kilroy SM, Lussier-Cushing M, Sumner L, et al. Falls in the general hospital: association with delirium, advanced age, and specific surgical procedures. Psychosomatics 2009; 50:218-226.

56. Tohen M, Angst J. Epidemiology of Bipolar Disorder (2nd Ed). In: Tsuang MT, Tohen M, Editors. Textbook in Psychiatric Epidemiology. Hoboken: John Wiley \& Sons, 2002, p.427-444.

57. Möller-Leimkühler AM. Barriers to help-seeking by men: a review of sociocultural and clinical literature with particular reference to depression. J Affect Disord 2002;71:1-9.

58. Ghaemi SN, Sachs GS, Chiou AM, Pandurangi AK, Goodwin FK. Is bipolar disorder still underdiagnosed? Are antidepressants overutilized? J Affect Disord 1999;52:135-144.

59. Admiraal J, Reyners A, Hoekstra-Weebers J. Do cancer and treatment type affect distress? Psychooncology 2013;22:1766-1773.

60. Strong V, Waters R, Hibberd C, Rush R, Cargill A, Storey D, et al. Emotional distress in cancer patients: the Edinburgh Cancer Centre symptom study. Br J Cancer 2007;96:868-874.

61. Daly M. Psychological Impact of Surgical Procedures on Women. In: Sadock BJ, Kaplan MI, Freedman AM, Editors. The Sexual Experience. Baltimore: William \& Wilkins, 1976, p.3081-3113.

62. Hollender MH. A study of patients admitted to a psychiatric hospital after pelvic operations. Am J Obstet Gynecol 1960;79:498-503.

63. Andersen BL, Hacker NF. Treatment for gynecologic cancer: a review of the effects on female sexuality. Health Psychol 1983;2:203-211.

64. Miller K, Massie MJ. Oncology. In: Levenson JL, Editor. Textbook of Psychosomatic Medicine and Consultation-Liaison Psychiatry. (3rd Ed). Washington: American Psychiatric Association Publishing, 2018, p.625-658.

65. Watts S, Prescott P, Mason J, McLeod N, Lewith G. Depression and anxiety in ovarian cancer: a systematic review and meta-analysis of preva- 
lence rates. BMJ Open 2015;5:e007618.

66. Trask P, Paterson A, Riba M, Brines B, Griffith K, Parker P, et al. Assessment of psychological distress in prospective bone marrow transplant patients. Bone Marrow Transplant 2002;29:917-925.

67. Gil F, Grassi L, Travado L, Tomamichel M, Gonzalez JR, Southern European Psycho-Oncology Study Group. Use of distress and depression thermometers to measure psychosocial morbidity among southern European cancer patients. Support Care Cancer 2005;13:600-606.

68. Arvidsdotter T, Marklund B, Kylén S, Taft C, Ekman I. Understanding persons with psychological distress in primary health care. Scand J Caring Sci 2016;30:687-694. 